\title{
BMJ Open Quality Introduction of an electronic patient record (EPR) improves operation note documentation: the results of a closed loop audit and proposal of a team-based approach to documentation
}

\author{
Joanna Aldoori 주 , Naomi Drye, Mark Peter, Jenifer Barrie
}

To cite: Aldoori J, Drye N, Peter M, et al. Introduction of an electronic patient record (EPR) improves operation note documentation: the results of a closed loop audit and proposal of a team-based approach to documentation. BMJ Open Quality 2019;8:e000766. doi:10.1136/ bmjoq-2019-000766

Received 8 July 2019 Revised 7 October 2019 Accepted 21 October 2019
D) Check for updates

(C) Author(s) (or their employer(s)) 2019. Re-use permitted under CC BY-NC. No commercial re-use. See rights and permissions. Published by BMJ.

Department of General Surgery, Calderdale and Huddersfield NHS Foundation Trust, Huddersfield, West Yorkshire, UK

Correspondence to

Joanna Aldoori;

joannaaldoori@doctors.org.uk

\section{ABSTRACT}

An operation note is a medicolegal document. The Royal College of Surgeons (RCS) of England's Good Surgical Practice 2014 (GSP) sets out 19 points an operation note should include. This study aimed to assess if the introduction of an electronic patient record (EPR) improved the quality of general surgical operation notes. An annonymised retrospective case note review of general surgical operation notes was undertaken over five separate time periods. The first cycle consisted of periods 1 (prior to EPR implementation), 2 (1 week after EPR) and 3 (4 weeks after EPR). Period 4 was a reaudit 2 weeks after the initial results were presented at the local governance meeting. The cycle was then closed with period $5 ; 1$ year after EPR implementation. A comparison was across all 5 time periods for compliance with the RCS guidelines and with subanalysis of the individual categories. 250 operation notes were reviewed during five time periods. Compliance improved by almost $19 \%(p=0.0003)$ between periods 1 and 5 . Eleven of the 19 points (57.9\%) over the audit period achieved $100 \%$ compliance post-EPR compared to $0 \%$ prior. Poor compliance were noted in the categories of antibiotic use, venous thromboembolism prophylaxis and estimated blood loss (noting that these are often documented in the anaesthetic record and/or WHO checklist). EPRs do not guarantee compliance with GSP. We propose that GSP standards need to be updated to reflect the modernisation of medical records and a team-based approach with multimodality input sources would achieve better patient records and patient care.

\section{PROBLEM}

Patient's records are being modernised; the way they are used and stored has changed dramatically and continues to evolve as we work towards a paperless NHS by $2020 .{ }^{1}$ In 2014, the Royal College of Surgeons of England (RCS Eng) published 'Good Surgical Practice'2 (GSP) a set of standards for surgeons, including guidelines for how surgeons should document their work. For operation notes, this consists of a clear list of criteria and includes 19 points that they should include (see figure 1).
An operation note is a medicolegal document describing an operative procedure and provides a detailed outline of the procedure the patient has undergone, in addition to guidance for ongoing care and management.

The Calderdale and Huddersfield Foundation Trust (CHFT) is a moderately sized district general hospital across two sites delivering acute surgical services, specialist surgical services (breast, urology, vascular, colorectal and benign upper gastrointestinal surgey) and is also a tertiary referral bariatric centre.

On the 1 May 2017, CHFT implemented an electronic patient record (EPR) system overnight. This was on a trust level and consisted of the disappearance of the clinical notes trolley with paper files and the appearance of several computers in which to deliver and record patient care. In addition to multiple levels of strategic planning, all members of staff underwent at least an afternoon of training. All aspects of patient care: ward rounds, drug charts, fluid balance charts, nursing documentation and so on, took place on the EPR system (Cerner Melenium). With the exception of the anaesthetic record which continued to be recorded on paper and the WHO checklist, which was documented on a different Theatre Management Program (Bluespier).

The project aimed to achieve $100 \%$ compliance with RCS GSP recommendations for operation note documentation.

A closed loop retrospective audit was undertaken to assess to what degree the introduction of an EPR system had improved the quality of operation note documentation and compliance with RCS Eng GSP in the general surgical department of a district general hospital over a 1 year period. 


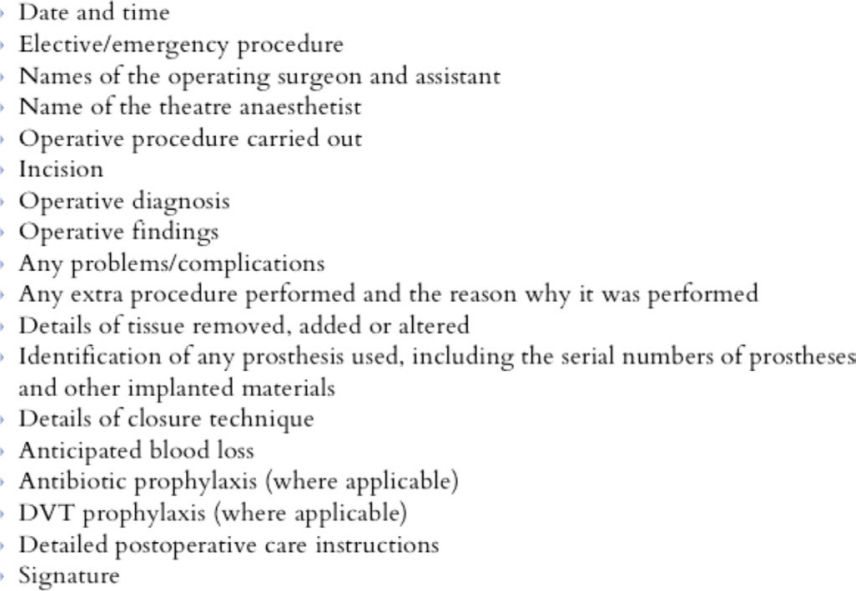

Figure 1 Taken from The Royal College of Surgeons of England's Good Surgical Practice (2014) lists what an operation note should include.

\section{BACKGROUND}

There is pre-existing literature demonstrating that electronic notes and operation-specific note templates improve operation note documentation as they reduce variability and prompt the surgeon completing the note to complete the data points in addition to being fully legible. ${ }^{3}$ Abbas et al found that a procedure-specific proforma for laparoscopic appendicectomy improved compliance with the RCS guideline from $66 \%$ to $94 \%$ $(\mathrm{p}<0.00001) .{ }^{4}$ Similarly, Barritt et al found that the introduction of detailed computerised proformas for patient undergoing hip hemi arthroplasties improved compliance from $58.7 \%$ to $92.8 \%(\mathrm{p}<0.1) .^{5}$

To our knowledge, there is very limited literature around the use of an EPR in improving compliance of operation notes with RCS Eng GSP.

\section{Measurement}

An anonymised retrospective case note review of general surgical operation notes was undertaken over five separate time periods. Five time periods were chosen in order to provide a reflective measure of improvement and use of the EPR system. The first cycle consisted of periods 1 (prior to EPR implementation, baseline measurement), 2 ( 1 week after EPR) and 3 (4 weeks after EPR). Period 4 was a reaudit 2 weeks after the initial results were presented at the local governance meeting. The cycle was then closed with period 5; 1 year after EPR implementation. A comparison was across all five time periods for compliance with the RCS guidelines and with subanalysis of the individual categories. Fifty consecutive, general surgery operation notes for both emergency and elective operations that took place under general anaesthetic, and in the main operating theatres were reviewed across each time period. Authors JA, ND and JB collected data, the general surgical team were not made aware of the audit periods in order to minimise the potential for bias.

A $100 \%$ compliance with the RCS guideline was expected. Statistical analysis was performed using a $2 \times 2$ contingency table and Fisher's exact test to calculate a two-tailed $p$ value. A $p$ value of $<0.05$ was considered statistically significant.

Baseline measurement found that overall compliance with GSP was $64.5 \%$. None of the data points reached full compliance. Only 1 (2\%) operation note was typed. Only $31(62 \%)$ operation notes included both the date and time of the procedure and 26 (52\%) included the name of the operation surgeon and assistant. This has major medicolegal implications.

\section{Design}

Though regular auditing of operation notes against GSP we aimed to observe how compliance could be improved by identifying specific data points were compliance was not achieved. After the first audit cycle, the results were presented to the entire general surgical department. The intervention was to raise awareness and education of department about the use of the EPR and operation note documentation. Following this, a reaudit was undertaken 2 weeks after this and a year after the EPR had been implemented.

The project team represented a full surgical firm as it consisted of: a foundation year one doctor, a core surgical trainee, a higher surgical trainee and a consultant surgeon

Using the EPR has been a steep learning curve and there has had to be additional training and use of EPR experts to educate the team as how best to use the system to serve patients effectively.

\section{Strategy}

PDSA cycle 1

We conducted an audit after the initial introduction of the EPR and found that it had improved operation note documentation dramatically. Compliance improved from $64.5 \%$ at baseline measurement to $83.5 \%$ at period 3,4 weeks after its introduction. Areas were there was lack of compliance was the name of the theatre anaesthetist, antibiotic and Deep Vein Thrombosis (DVT) prophylaxis. This was highlighted and awareness raised to the general surgical department at the clinical governance meeting.

\section{PDSA cycle 2}

A further reaudit was undertaken 2 weeks after the clinical governance meeting. Compliance plateaued at $83.5 \%$ at period 4, 2 weeks after the clinical governance meeting and $83.4 \%$ at period 5 , a year after the implementation of EPR. Data points which continued not to fulfil compliance included: anticipated blood loss, antibiotic and DVT prophylaxis in addition to the name of the theatre anaesthetist. Raising the possibility that perhaps the measurement tool, GSP, for operation notes needs updating to reflect the use of an EPR and a team approach to documentation.

\section{RESULTS}

A total of 250 operation notes were reviewed over the 5 separate time periods, which represented a complete audit cycle. 
Table 1 Demonstrating the results across the audit cycle

No of notes with data points completed (\%)

$2 \times 2$

Contingency

table using

$\%$ Change Fisher's exact

from period test between

Data point

Date and time

Period 1

Period 2

Period 3

Period 4

Period 5

1 to 5

period 1 and 5

Elective/emergency procedure

Names of the operating surgeon

and assistant

Name of the theatre

anaesthetist

Operative procedure carried out

Incision

Operative diagnosis

Operative findings

Any problems/complications

Any extra procedure performed

and the reason why it was

performed

Details of tissue removed, added or altered

Identification of any prosthesis

used, including the serial

numbers of prostheses and

other implanted materials

Details of closure technique

Anticipated blood loss

Antibiotic prophylaxis (where

applicable)

DVT prophylaxis (where

applicable)

Detailed postoperative care

instructions

Signature

$31(62)$
$2(4)$
$26(52)$

50 (100)

50 (100)

50 (100)

50 (100)

$50(100)$

50 (100)

50 (100)

50 (100)

$+38$

0.0001

50 (100)

50 (100)

50 (100)

$50(100)$

$+96$

0.0001

$50(100)-50(100)+50)$

$43(86)$

$25(50)$

$28(56)$

$29(58)$

$30(60)$

$-26$

0.0063

46 (92)

50 (100)

$50(100)$

50 (100)

\section{0 (100)}

$+8$

0.1175

46 (92)

$42(84)$

50 (100)

50 (100)

50 (100)

$+8$

0.1175

$46(92)$

49 (98)

$50(100)$

50 (100)

50 (100)

0.1175

49 (98)

50 (100)

$50(100)$

50 (100)

0.1175

$20(40)$

$18(36)$

21 (42)

$16(52)$

$24(48)$

0.5459

46 (92)

$50(100)$

50 (100)

$50(100)$

$50(100)$

0.1175

39 (78)

49 (98)

48 (96)

47 (94)

46 (92)

$+14$

0.0905

48 (96)

47 (94)

47 (94)

48 (96)

$+4$

0.6777

\begin{tabular}{cccccll}
$46(92)$ & $49(98)$ & $49(98)$ & $47(94)$ & $49(98)$ & +6 & 0.3622 \\
$0(0)$ & $18(36)$ & $13(26)$ & $10(20)$ & $14(28)$ & +28 & 0.0001 \\
$17(35)$ & $19(38)$ & $18(36)$ & $25(50)$ & $21(42)$ & +7 & 0.5368 \\
$20(40)$ & $17(34)$ & $19(38)$ & $23(46)$ & $11(22)$ & -18 & 0.0828 \\
$46(92)$ & $49(98)$ & $50(100)$ & $49(98)$ & $49(98)$ & +6 & 0.3622 \\
$46(92)$ & $50(100)$ & $50(100)$ & $50(100)$ & $50(100)$ & +8 & 0.1175 \\
$1(2)$ & $50(100)$ & $50(100)$ & $50(100)$ & $50(100)$ & +98 & 0.0001 \\
$64.5 \%$ & $82.3 \%$ & $83.5 \%$ & $83.5 \%$ & $83.4 \%$ & +18.9 & 0.0003 \\
\hline
\end{tabular}

Overall compliance

$46(92)$
$1(2)$
$\mathbf{6 4 . 5 \%}$

Areas shaded in grey represent $100 \%$ compliance.

Data points in bold did not achieved full compliance throughout the audit period.

With the exception of one operation note $(2 \%)$ the majority of operation notes in period 1 were hand written.

From period 1 to 5, overall compliance improved by almost 19\% (p 0.0003). The results across all time periods are presented in the table 1 and figure 2.

Eleven of the 19 points (57.8\%) achieved $100 \%$ compliance over the study period. The points that showed the greatest improvement were data and time $(62 \%-100 \%$, $+38 \%, \mathrm{p}=0.0001$ ), whether the procedure was emergency or elective $(4 \%-100 \%,+96 \%, \mathrm{p}=0.0001)$, names of the operating surgeon and assistant $(52 \%-100 \%,+48 \%$, $\mathrm{p}=0.0001)$ and that the notes were all typed $(2 \%-100 \%$, $+98 \%, \mathrm{p}=0.0001)$.

Areas where there was no or limited improvements were: name of theatre anaesthetist $(86 \%-60 \%,-26 \%$, $\mathrm{p}=0.00063)$, problems or complications $(40 \%-48 \%$,
$+8 \%, \mathrm{p}=0.5459)$, in addition to antibiotic $(35 \%-42 \%$, $+7 \%, \mathrm{p}=0.5368)$ and DVT prophylaxis $(40 \%-22 \%,-18 \%$, $\mathrm{p}=0.0828)$.

\section{Lessons and limitations}

The results of this closed loop audit demonstrate that an EPR improved compliance with the RCS GSP guideline. Compliance improved in all of the 19 points over the audit period except for the name of the theatre anaesthetist where compliance was greater prior to EPR.

Prior to the introduction of EPR, operation notes were mostly handwritten and enclosed in the theatre pathway. These notes were then scanned on to the separate electronic document management application; the application used was electronic document management system (EDMS). Notes were often difficult and time-consuming 


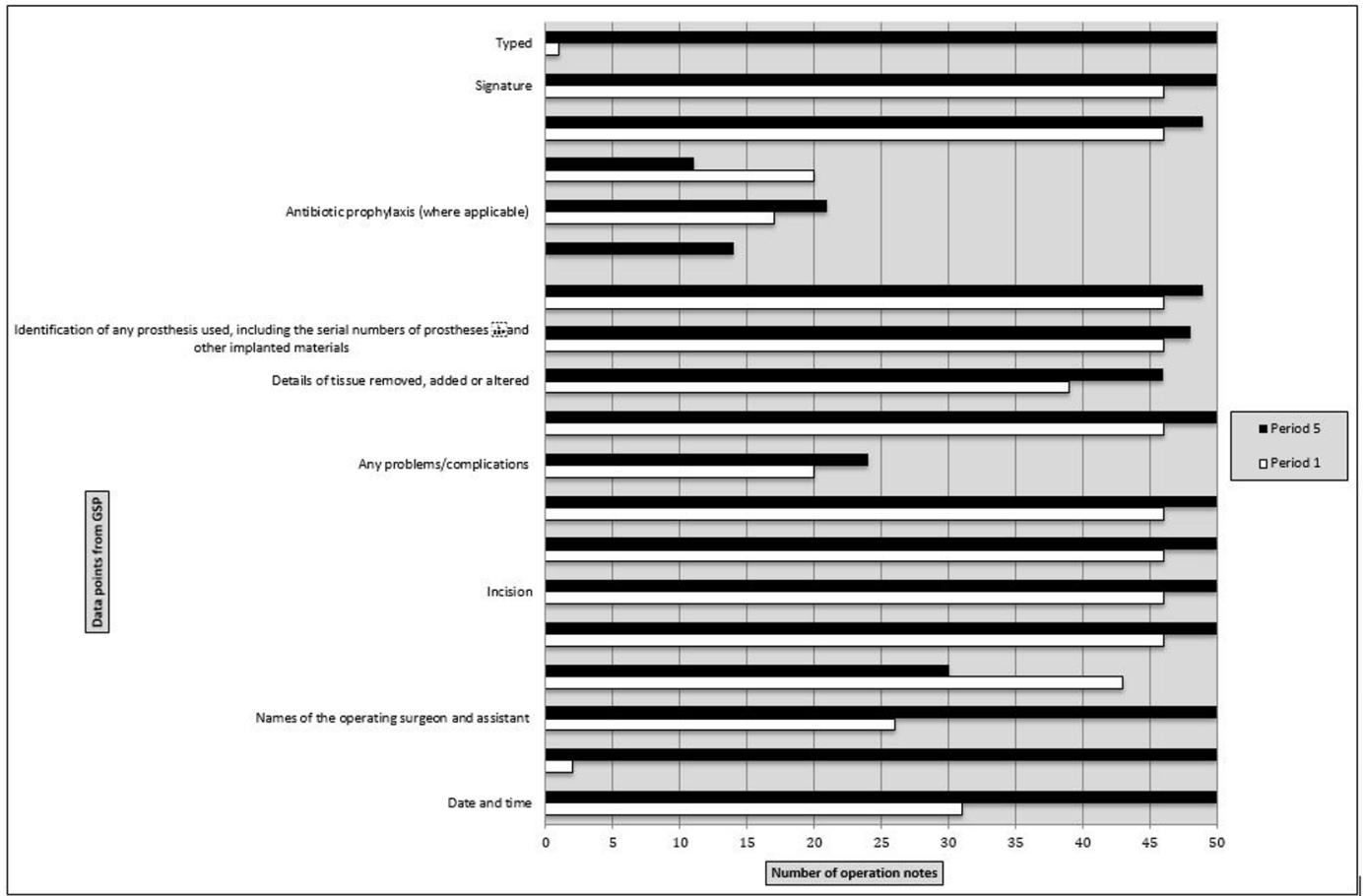

Figure 2 Bar chart illustrating compliance with each data point between period 1 and period 5.

to locate, as it required the viewer to click through pages of paper scanned on to a computer and they were often mislabeled.

Identifying an operative record is a more intuitive process using the EPR system and notes are immediately available in the patient records without requiring further access to an electronic document management/scanning application such as EDMS. Patient care can be delivered more efficiently as notes can be found with greater ease, this is particularly important for patients who are acutely unwell or are readmitted following an operative procedure with a potential complication.

EPR systems allows the immediate coding of operations by the clinician, this clearly poses a benefit in that coding is undertaken directly by clinician and therefore is more likely to be correct.

Limitations include the retrospective nature of the audit. We were unable to locate four operation notes, during period 1 which took place prior to EPR, on the electronic document management application. This may be because the operation notes were not scanned on to the system, or the hard copy may have been lost. This poses a medicolegal implication for a number of reasons. The sample was generated from 50 consecutive general surgery operation notes and therefore there is heterogeneity within the samples. Heterogeneity of the data is a weakness of the study; however, the study aimed to assess how the introduction of an EPR system improved operation note documentation on a practical level and therefore the heterogeneity, in the types of operations and authors of the operation notes, portrays the practicality of an EPR to document operations. Of course compliance could have improved by restricting the sample to a single operation such as laparoscopic appendicectomy and creating mandatory fields or a laparoscopic appendicectomy template; however, there is a real danger that we reduce an operation note to a tick box or copy and pasting exercise where in reality the surgeon does not take the time to document correctly. In addition, this approach to documentation makes it difficult for some emergency operations, which have a variety of findings, operative interventions and the need more than one surgical specialty involvement to be documented appropriately. No fields are mandatory on the electronic operation note, and although there are subject headings within the operation note, the user is not required to fill every field in order to publish the note. By making fields mandatory, we could ensure full compliance with the RCS guideline; however, we do not feel this is the best way of achieving compliance or accurate documentation. What our study has highlighted is that the process in which we document operations needs updating to reflect an EPR.

We have found that although users of the EPR system were given a minimum of an afternoon of training, much of what is practiced is learnt on the job and this continues to be a dynamic process.

Hundred per cent compliance was met with some data points following introduction of EPR, these included: date and time, whether the procedure was elective or emergency, the name of the operative procedure carried out and the surgeon's signature writing the note. This was likely because these fields are auto filled or have ease of being filled on the system by a tick box, such as date and time and whether the operation is elective or an emergency. Therefore, $100 \%$ compliance is virtually guaranteed for these data points. 
Although compliance improved significantly from period 1, prior to EPR and period 5, a year after EPR, compliance plateaued and did not significantly increase between periods 2 and 5 despite a period of education and a time for users to adjust to EPR. This could be due to the way in which the operation note is written, usually away from the operating theatre and in the operating theatre coffee room, therefore important information such as the name of the anaesthetist, serial numbers of any prosthetics used, DVT and antibiotic prophylaxis in addition to estimated blood loss are not always recorded in operation notes. This information is, however, recorded as part of the theatre pathway, either in the anaesthetic chart or nursing documentation, therefore we would suggest that there should be a team approach to some aspects of the GSP data points to avoid duplication and ensure accurate documentation, this could take place as part of the WHO time out, before the whole team disperse.

This study has shown that an EPR improves operative note documentation in certain data points but does not provide a guaranteed way to ensure documentation meets the RCS guidelines. It raises the possibility of the overall effectiveness of the measurement tool GSP which was last updated in 2014. GSP should be updated to reflect the use of EPRs and the team approach to documentation.

\section{CONCLUSION}

Procedure-specific and operation note templates have been shown to improve operation note compliance with GSP; however, there is a potential for an operation not to become a tick box exercise and may not accurately document the procedure. The EPR improved operation not documentation but it there were specific data points that did not achieve compliance such as antibiotic and DVT prophylaxis, anticipated blood loss and the name of the theatre anaesthetist. This project specifically assessed the use of a new system for documentation. We believe that our current guidelines for documentation need updating to reflect the use of an EPRs with a team-based approach to documentation.

Surgeons do not work in isolation and there is increasing literature on the importance of team-based care $^{67}$ and how a team-based effort improves overall quality of care. Individual effort is often not enough as there are factors limiting the individual that are beyond their control. The results of this audit cycle show that specific aspects of operative note documentation such as: the name of theatre anaesthetist, problems or complications, estimated blood loss in addition to antibiotic and DVT prophylaxis were poor. We would suggest that GSP and operative notes should be updated to reflect team-based care. We propose this could be achieved through a multidisciplinary integrated theatre record, populated by specific members of the theatre team responsible for specific aspects of the patients care during their journey through the operating department. For example, anticipated blood loss should be estimated by the whole surgical team and not solely by the operating surgeon who glances at the suction at the end of the case. It is the shared responsibility of the whole team, the scrub nurse who gives the swabs to the circulator to measure the volume, the anaesthetist who monitors the bleeding throughout the case and transfuses appropriately. We believe that by documenting the patient's care throughout the operating department using this collaborative approach would improve patient care and outcomes.

To our knowledge, there are no current standards or guidelines for operation note documentation using an EPR. GSP was published in 2014 and we propose an update to these guidelines to reflect the use of EPRs and the team-based approach to caring for the surgical patient throughout the operating department.

EPRs improve the legibility and accessibility of operation notes but are not a guarantee of comprehensive operation notes and compliance with The RCS 2014 GSP. We propose that GSP standards need to be updated to reflect the modernisation of medical records and a teambased approach with multimodality input sources would achieve better patient records and patient care.

Contributors JA initiated the project, performed the data collection, analysis and writing of the manuscript. ND assisted in the data collection and analysis, and approved the final manuscript. MP made critical revisions and approved the final manuscript. JB assisted in the data collection and analysis, made critical revisions and approved the final manuscript.

Funding The authors have not declared a specific grant for this research from any funding agency in the public, commercial or not-for-profit sectors.

Competing interests None declared.

Patient consent for publication Not required.

Provenance and peer review Not commissioned; externally peer reviewed.

Data availability statement All data relevant to the study are included in the article.

Open access This is an open access article distributed in accordance with the Creative Commons Attribution Non Commercial (CC BY-NC 4.0) license, which permits others to distribute, remix, adapt, build upon this work non-commercially, and license their derivative works on different terms, provided the original work is properly cited, appropriate credit is given, any changes made indicated, and the use is non-commercial. See: http://creativecommons.org/licenses/by-nc/4.0/.

\section{ORCID iD}

Joanna Aldoori http://orcid.org/0000-0002-5695-0000

\section{REFERENCES}

1 Parkin E. Briefing paper number 07572, 25 April 2016 a paperless NHS: electronic health records. house of commons library, 2016. Available: researchbriefings. parliament.uk/ResearchBriefing/Summary/ POST-PN-0519\#fullreport [Accessed 05 Aug 2018].

2 Royal College of Surgeons of England. Good surgical practice. RCS, 2014.

3 Ghani Y, Thakrar R, Kosuge D, et al. 'Smart' electronic operation notes in surgery: an innovative way to improve patient care. Int J Surg 2014;12:30-2.

4 Abbas SH, Singh S, Sundran R, et al. A thorough note: does a procedure-specific operation note proforma for laparoscopic appendicectomy improve compliance with the Royal College of Surgeons of England guidelines? Int J Surg 2016;2:1-5.

5 Barritt AW, Clark L, Cohen AMM, et al. Improving the quality of procedure-specific operation reports in orthopaedic surgery. Ann $R$ Coll Surg Engl 2010;92:159-62.

6 Dillon PW, Paz HL. Team-Based surgical care: an important role for academic health centers. JAMA Surg 2014;149:999-1000. 
